\title{
Silent reintroduction of wild-type poliovirus to Israel, 2013 - risk communication challenges in an argumentative atmosphere
}

E Kaliner (ehud.kaliner@moh.health.gov.il)1, J Moran-Gilad ${ }^{1,2}$, I Grotto ${ }^{1,2}$, E Somekh ${ }^{3,4}$, E Kopel ${ }^{1}$, M Gdalevich ${ }^{2,5}$, E Shimron $^{6}$,

Y Amikam ${ }^{6}$, A Leventhal ${ }^{6}$, B Lev 6 , R Gamzü,6

1. Public Health Services, Ministry of Health, Jerusalem, Israel

2. Faculty for Health Sciences, Ben-Gurion University of the Negev, Beer-Sheva, Israel

3. Paediatric Infectious Diseases, Wolfson Medical Center, Holon, Israel

4. Sackler Faculty of Medicine, Tel-Aviv University, Tel Aviv, Israel

5. South District Health Office, Ministry of Health, Beer Sheva, Israel

6. Ministry of Health, Jerusalem, Israel

Citation style for this article:

Kaliner E, Moran-Gilad J, Grotto I, Somekh E, Kopel E, Gdalevich M, Shimron E, Amikam Y, Leventhal A, Lev B, Gamzu R. Silent reintroduction of wild-type poliovirus to Israel, 2013 - risk communication challenges in an argumentative atmosphere . Euro Surveill. 2014;19(7):pii=20703. Available online: http://www.

eurosurveillance.org/ViewArticle.aspx?Articleld=20703

Israel has been certified as polio-free by the World Health Organization and its routine immunisation schedule consists of inactivated poliovirus vaccine (IPV) only. At the end of May 2013, the Israeli Ministry of Health $(\mathrm{MOH})$ has confirmed the reintroduction of wild-type poliovirus 1 into the country. Documented ongoing human-to-human transmission necessitated a thorough risk assessment followed by a supplemental immunisation campaign using oral polio vaccine (OPV). The unusual situation in which ongoing poliovirus transmission was picked up through an early warning system of sewage monitoring without active polio cases, brought about significant challenges in risk communication. This paper reviews the challenges faced by the $\mathrm{MOH}$ and the communication strategy devised, in order to facilitate and optimise the various components of the public health response, particularly vaccination. Lessons learned from our recent experience may inform risk communication approaches in other countries that may face a similar situation as global polio eradication moves towards the 'End game'.

\section{Background}

Israel has been certified as free of poliovirus by the World Health Organization (WHO) along with the entire WHO European Region in 2002 and has been using an inactivated polio vaccine (IPV)-only immunisation strategy since 2005, as most European Union (EU) countries do.

At the end of May 2013, the Israeli Ministry of Health $(\mathrm{MoH})$ confirmed the reintroduction of wild-type poliovirus 1 (WPV 1 ) into Israel following its detection in routine environmental surveillance of poliovirus in the sewage system near the town of Rahat, southern Israel. Enhanced surveillance for clinical cases did not detect any cases of paralytic poliomyelitis, most likely due to high vaccine coverage rate of the population, above $95 \%$ before WPV 1 has first been detected at end of May, and above $98 \%$ after the inactivated poliovirus vaccine (IPV) catch-up campaign that was undertaken during June and July, evident from the computerised National Vaccination Registry [1]. On 5 August, following thorough epidemiological and virological investigation and the recommendation of an invited WHO mission, the $\mathrm{MoH}$ decided to conduct a supplemental immunisation activity (SIA) to vaccinate all children aged from 0 to 9 years in the southern region of the country (Southern District) who had not received oral polio vaccine (OPV) in the past, with a bivalent oral polio vaccine (bOPV). Two weeks later, following continuous circulation of WPV1, the SIA was extended to cover the entire country [1].

This has been a unique and unprecedented situation and a public health challenge within the global polio eradication efforts. To our knowledge, this has been the first case of detection of WPV by an early warning system of environmental surveillance and not through presentation of polio cases, into a 'polio-free' country that uses 'IPV only' as its routine vaccination regimen [2]. This special situation has made the decision to deploy an SIA with OPV complex in terms of risk communication. This paper reviews the challenges faced by the $\mathrm{MoH}$ and the communication strategy formulated in order to facilitate and optimise various components of public health response, particularly vaccination, and lessons learned at the national level.

\section{Risk communication challenges}

From the onset, it became clear that risk communication will play a crucial role in building trust among the public and professional stakeholders. Therefore, the Ministry's communication and media experts were full members of the national outbreak control team. The 
$\mathrm{MoH}$ identified challenges related to risk communication as:

- communicating the risk associated with a silently circulating WPV1 in a highly immunised community;

- communicating the decision and the rationale behind it (need and urgency) for public health action, particularly an SIA, despite the lack of paralytic polio cases;

- communicating the advantage of an SIA using OPV to halt WPV 1 circulation, in a community already highly immunised with IPV;

- communicating the benefits of an SIA using OPV taking into consideration individual (prevention of viral shedding), public (eradication of the virus and protection of contacts) and global (polio eradication) health aspects;

- communicating the safety and risks of OPV in general and bOPV in particular;

- communicating the rationale of reintroducing OPV after its use was stopped in 2005 .

\section{Communication strategy during early phase} of the investigation

In the WHO published guidelines for risk communication in an outbreak situation $[3,4]$, the basic principles are trust, early announcement, transparency, 'the public' and planning. The first three components are difficult to separate. The main goal of communication management is building and maintaining public trust. Routine childhood vaccination programmes in Israel are not obligatory and are generally perceived as having a good reputation, as reflected by the high rate of coverage for all vaccines (over 95\%) (unpublished data).

An important tool in maintaining trust is transparency to avoid potential accusations of concealing information by decision makers. This requires that the public receives full information from an official health authority. In accordance with this approach, within one day, after the confirmation of WPV 1 circulation in the country, a report using information available to the $\mathrm{MoH}$ was made public [5].

The rationale underlying early announcement was to provide information to the media and establish the $\mathrm{MoH}$ as the most reliable source while aiming at preventing media reports of rumours and speculations from emerging and spreading, especially via social media [6].

Similarly, the media and the public were informed about any new data generated from environmental surveillance and population surveys and about subsequent public health response, in a timely manner. Except for one instance where preliminary laboratory results were leaked to the media, all information held exclusively by the $\mathrm{MoH}$, was made public via official media briefings. To prevent a situation where the public receives information before healthcare professionals, a major effort was undertaken to ensure that all briefing materials intended for both professionals and lay public were prepared simultaneously and distributed first to healthcare professionals and $\mathrm{MoH}$ employees and immediately afterwards to the public.

Communication strategy in preparation for the supplemental immunisation activity

Following consultations with WHO experts during their mission to Israel, preparations for a SIA began as part of a coordinated public health response plan. An integral part of preparedness included a comprehensive communication plan. The Government Advertising Bureau was recruited for this task and a decision was made to design two campaigns - one that would focus on hygiene and be launched before starting the SIA, and another one that would focus on vaccination. As a complementary measure, the MoH sought counselling from a commercial strategic consulting firm to prepare the communication plan and to design key messages for the public. Moreover, the firm was asked to advise on management and monitoring of communication in electronic media, in particular social networks, that were identified during the 2009 influenza $A\left(\mathrm{H}_{1} \mathrm{~N}_{1}\right)$ pandemic as having an have an important impact on public opinion $[7,8]$.

In the planning process, we identified a number of potentially critical points. The first and most important was to achieve consensus amongst the medical community regarding the need for a SIA [9]. During the 2009 pandemic in Israel, vaccination campaigns faced difficulties as a sizeable number of physicians did not support or opposed influenza vaccination [10]. In the presented incident, many opinion-leading physicians employed by health organisations outside the $\mathrm{MoH}$, including paediatricians, family physicians, infectious disease and neurology consultants, were fully engaged in the $\mathrm{MoH}$ decision regarding vaccination. This was achieved by discussions and briefings of chairs of national medical societies, national medical councils and the Israeli Medical Association and information delivery to the entire medical community. Points of contact at the MoH Public Health Services were established to provide feedback and consultation to frontline physicians [11]. In weeks after the decision to deploy the SIA, dozens of conferences were held by $\mathrm{MoH}$ senior representatives in every hospital in the country and in every region. At the political level, the Israeli Government received periodic briefings and the Health Minister was engaged in key decisions. These measures achieved an almost end-to-end consensus amongst the medical community, to the point where local 'anti-vaccine' movements had to seek support from known anti-vaccine doctors in other countries e.g. in the United States and India [12].

A notable example of engagement was the reaction of primary care paediatricians in Israel. Paediatricians' attitudes were monitored by their inputs into the paediatric professional electronic network (IPRONET) that is used by 500 paediatricians across Israel and therefore 
served as an indicator to their viewpoints. IPRONET communications as well as small focus groups held prior to the campaign revealed that most paediatricians declared that they were neither going to recommend OPV to their patients, nor vaccinate their own children, due to the perceived low risk-benefit ratio of OPV. This was mitigated by seven informal information papers and daily responses to frequently asked questions (FAQ) by senior paediatricians in their network during the SIA together with direct formal email briefings from the $\mathrm{MoH}$ regarding the status of WPV 1 circulation and rationale for the SIA, using email contact lists provided by medical organisations and associations. Continuous monitoring of network postings by the site coordinators documented a gradual shift from scepticism to enormous support of the campaign (E Somekh, personal communication, 15 August 2013).

The second critical point was that ideological antivaccination groups were expected to take advantage of the situation and attack vaccines in general and the 'new' polio vaccination with bOPV in particular. It was decided not to confront those movements head-on, but to prevent them from negatively influencing the general public as at that point in time, only $55 \%$ of parents said they would vaccinate their children against polio although $80 \%$ said they do believe an $\mathrm{MoH}$ action is needed (Government Advertising Bureau commissioned survey, Y Amikam, personal communication, 10 July 2013). Indeed, even before the campaign was launched, antivaccinists started advocating against it, especially via electronic media.

The third point was the rationale for the SIA using OPV was complicated to deliver and it was even harder to motivate the public to take action and get vaccinated. An SIA had also been carried out in the neighbouring Egypt a few months earlier due to WPV circulation detected through environmental surveillance. However, a profound difference was that Egypt has OPV in routine childhood immunisation programme [13]. The idea of reintroducing a live attenuated vaccine that had been abandoned nearly a decade ago was difficult to understand.

Not only did the fact that there were no paralytic cases make the decision to launch a SIA difficult for policy makers, it certainly also altered the perception of risk among the public. In this respect, the SIA seemed to have become a 'victim' of the success of the environmental surveillance, as much as vaccination has become a victim of its own success [14].

The fact that OPV was to be given to children who were already vaccinated with IPV and hence, protected from paralysis in the event of exposure to WPV1, caused much hesitation amongst parents who subsequently decided not to pursue vaccination with OPV due to the perception of the risk for paralytic polio and risk associated with vaccine administration. Many parents felt that OPV was a 'social' vaccine that builds on herd immunity and compensates for the small percentage of the population that has not received IPV, and felt that administering OPV to their IPV-vaccinated children is merely altruistic. Mindful of that, the message to the public was that the vaccine will protect their family members and close friends and not just the individual or the 'environment' or 'society'. Accordingly, the main slogan of the campaign was 'Just two drops and the family is protected from the risk of polio' (Figure 1).

In the information era, the number of sources of information is immense and as a result, the $\mathrm{MoH}$ had to interact or be present in all of them in order to deliver valid information and ensure accessibility of the public to information. The use of a variety of communication measures is an important principle in risk communication plans [8]. Examples of communication channels used by the $\mathrm{MoH}$ in the incident under discussion included (i) a new designated official polio $\mathrm{MoH}$ website, (ii) an existing official Facebook interface, (iii) an existing national call centre involving over 20 of $\mathrm{MoH}$ staff for several weeks reinforcing polio communications (agreed key messages and answering FAQ), (iv) print media (national and sectorial in various languages) and (v) electronic journalism, including, for the first time, various social networks, forums and blogs. Israel has several communities whose first language is not Hebrew but e.g. Arabic, Russian and Tigrigna and information was provided in all common languages spoken in Israel. The relationship and the flow of information between the elements of communication are described in Figure 2.

\section{FIGURE 1}

Campaign slogan for supplemental immunisation activity with bivalent oral polio vaccine during silent reintroduction of wild-type poliovirus 1, Israel, 2013

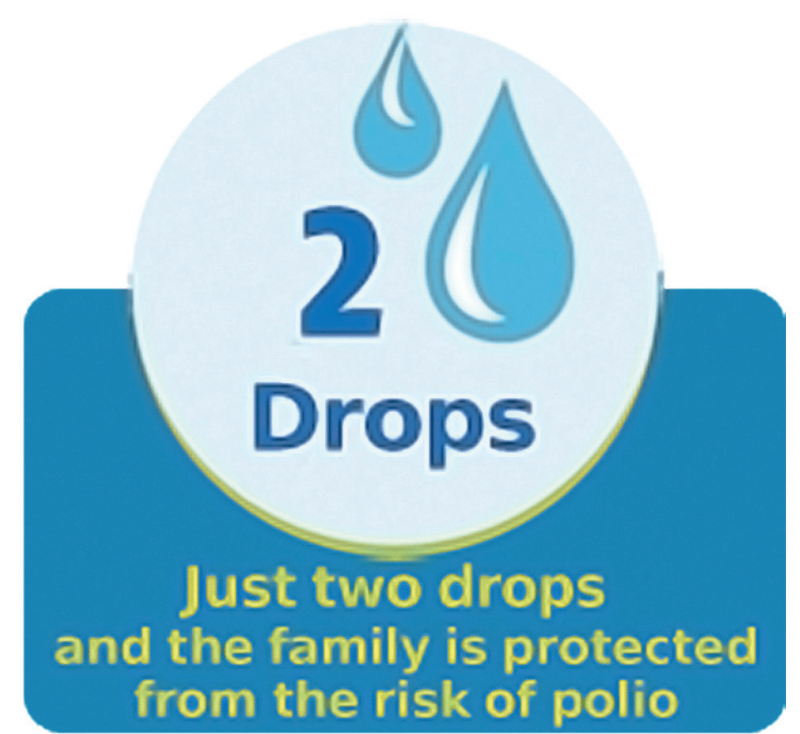




\section{Communication strategy during the SIA}

The SIA was carried out in two phases. First it started in the Southern District which appeared as the epicentre of WPV 1 introduction. After two weeks, following ongoing WPV 1 activity, the full national phase was launched.

There has been an obvious increase in Internet use for gathering information compared to similar events in the past. Browsing activity monitoring of the official $\mathrm{MoH}$ polio campaign website is described in the Table. Notably, most browsing activities were up to three minutes long, and involved single page views ('bounces') in over half of cases.

The $\mathrm{MoH}$ decided on a massive and continuous presence of public health professionals who had undergone ad hoc training to answer questions, eliminate ambiguity and rectify disinformation through online and traditional media. This approach proved effective in motivating people to comply with medical recommendations as previously described [15].

One of the problems that arose from deployment of multiple responders, however, was occasional lack of uniformity in messages. Rare cases of contradicting messages (almost always concerning minor issues) were picked up by vaccination opponents and used for attacking the campaign and questioning the $\mathrm{MoH}$ credibility. Frequent briefing of professional responders aimed at preventing such situations, but uniform messaging remains a challenge for management of future incidents. By the time the SIA was launched, the continuous sharing of information and transparency had harnessed almost all health journalists to support the SIA and its objectives.

\section{FIGURE 2}

Information flow during silent reintroduction of wild-type poliovirus 1 and supplemental immunisation activity with bivalent oral polio vaccine, Israel, 2013

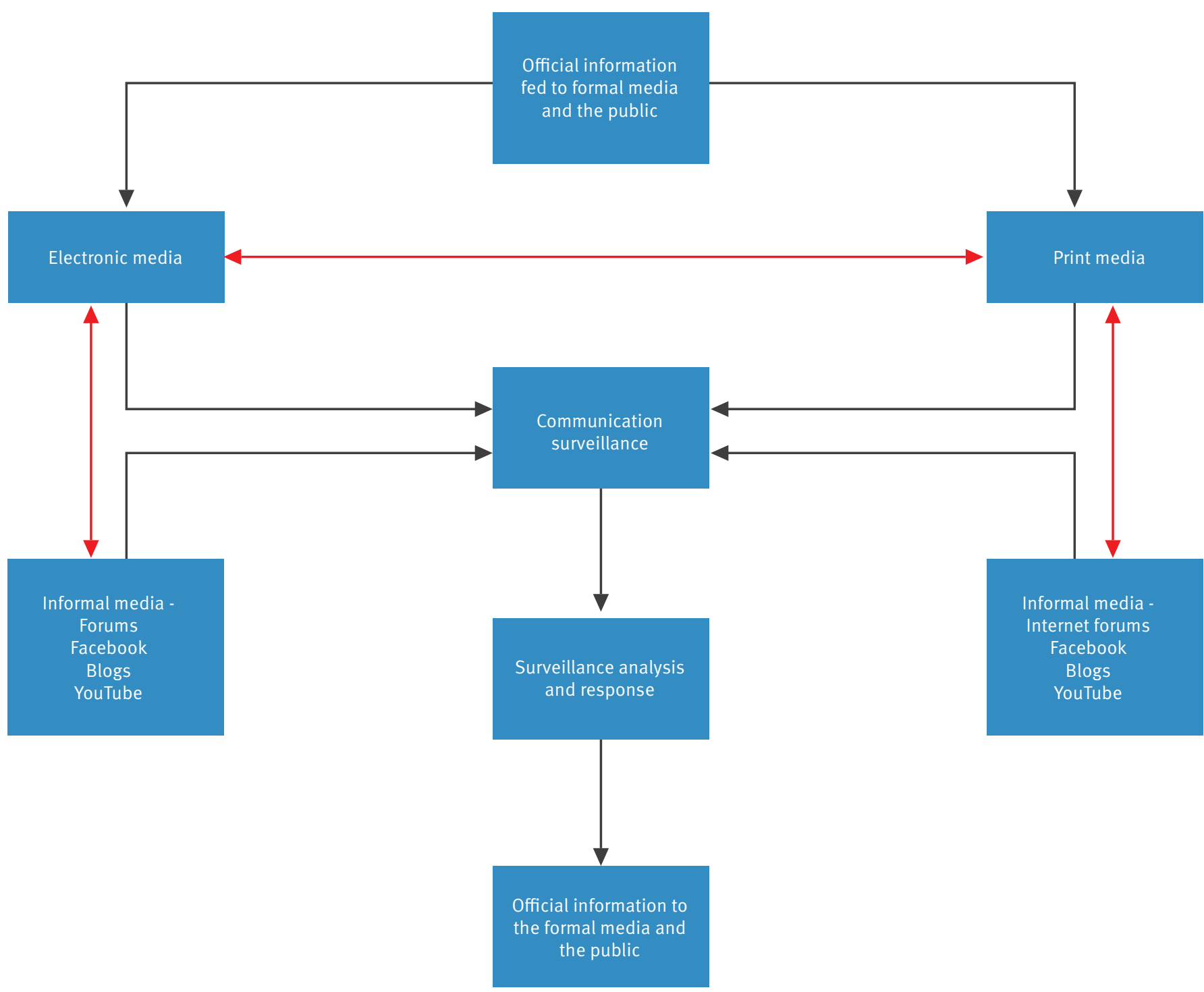


During the campaign, public figures were recruited to support it, including members of the Knesset, the Israeli parliament, and the president of Israel. $\mathrm{MoH}$ physicians were photographed while vaccinating their family members as reassurance to inquiries from members of the public who questioned whether health professionals practise what they preach. At the individual level, phone calls and SMS messages to parents who did not vaccinate their children were used to provide information as needed.

Monitoring of the media was performed throughout the SIA in order to identify the 'hot' issues in the public debates and comment on them e.g. vaccine safety, necessity, side effects, etc. A significant decrease in public interest was observed over time due to 'fatigue' from the 'polio issue', summer leave and Jewish High Holidays in September, as well as regional security concerns. Despite that 'fatigue', fundamental vaccine opponents continued to object to the SIA mostly through certain known anti-vaccine websites and in an active approach $\mathrm{MoH}$ responders started conversations over the Internet.

As mentioned above, one of the 'hot' issues was vaccine safety. The public questioned many aspects of OPV such as clinical trial data and evidence base, data from previous OPV use and the risk of vaccine associated paralytic polio (VAPP). One of the arguments of the vaccine opponents was that the $\mathrm{MoH}$ yielded to international pressure from $\mathrm{WHO}$ and other international health authorities as part of a global campaign and not a local need. The risk perception of the vaccine was enhanced and public anxiety was directed towards VAPP rather than the risk from WPV 1 circulation. In

\section{TABLE}

Number of entries to Ministry of Health campaign website 'Just two drops'a by topics, Israel, 4 August-16 September 2013

\begin{tabular}{|l|l|}
\hline Web page & $\begin{array}{l}\text { Visitors } \\
\text { (number) }\end{array}$ \\
\hline Main homepage & 277,290 \\
\hline Poliovirus - general information & 94,218 \\
\hline Frequently asked questions (FAQ) & 194,897 \\
\hline Vaccination centres (map, addresses, working hours) & 170,646 \\
\hline The disease and the vaccine & 28,216 \\
\hline Latest updates & 19,604 \\
\hline Guidelines for immunocompromised patients ${ }^{\text {b }}$ & 7,873 \\
\hline Hygiene and hand washing & 5,799 \\
\hline Vaccination coverage ${ }^{\text {b }}$ & 11,091 \\
\hline Spokesman announcements & 14,745 \\
\hline Routine childhood vaccines & 9,263 \\
\hline Routine childhood vaccines (in detail) & 11,190 \\
\hline Total & $\mathbf{8 4 4 , 8 3 2}$ \\
\hline
\end{tabular}

a URL: http://www.health.gov.il/English/Topics/Vaccination/ two_drops/Pages/default.aspx.

b Launched on 20 August 2013. order of mitigate those concerns, data from post marketing safety surveillance, the package insert of the vaccine, laboratory testing results of the specific bOPV lots used and clinical trials done with the vaccine were published on the official $\mathrm{MoH}$ website and questions in social media answered by a dedicated MoH specialist.

\section{Lessons learned from polio communication strategy}

By mid-October, more than 900,000 children of 1.2 million candidates were vaccinated. Seemingly, this coverage rate had a favourable epidemiological effect - as judged by the dramatic reduction in prevalence of WPV1 detected during environmental surveillance and field surveys - but was it successful from a risk communication point of view? How can health authorities improve compliance during SIAs?

First, health authorities need to assess their methods of communication before and during an outbreak of infectious disease [16]. This is certainly true in a major event that lacks concurrent morbidity. Risk communication in relation to vaccine-preventable diseases (VPDs) involves certain unique aspects, especially those related to the safety, efficacy and effectiveness of vaccination, and social factors such as anti-vaccination movements, conspiracy theories and ethical discourse surrounding equality, autonomy and mutual guarantee. The current silent transmission of the poliovirus involved communication challenges related to VPDs in general, but also specific challenges related to public health policy built on early warning signals, perception of disease risk in a vaccinated population and reintroduction of a 'forgotten' pathogen and vaccine.

Second, the importance of the Internet as key media was acknowledged and emphasised in this campaign. However, one cannot abandon traditional media since not everyone has Internet access. Billboards, radio, television and traditional mail messages have proven particularly useful among specific sectors with limited Internet use. This finding is in line with results obtained in a recent EU-wide opinion poll (Eurobarometer), suggesting that television is the main source of information on developments in science and technology, followed by newspapers and websites [17]. Another example is the use of the $\mathrm{MoH}$ call centre by the public which reflected public interest and concern over time (Figure 3).

Third, reliable information on vaccines, should preferably be made available routinely and not only when an SIA is contemplated. Such pro-vaccine sites may include narratives and stories of patients or family members thereof who fell ill with VPDs in order to have more impact. It is important to work on the image of the health authority as a credible source of information and judicious decision maker, throughout the year, so that the level of trust in the information provided will be high during public health emergencies. 
Health communication interventions can be strengthened through the adoption of a more holistic ecological model of people and their health-related behaviours analysed in the context of larger social, economic, political, and cultural forces [18] .Local stakeholders demand local content for their information feed and messages from a trusted local leader are the most superior forms of communication [19]. Israel is characterised by a heterogeneous population, consisting of social, ethnic or religious communities with distinctive cultural characteristics, occasionally involving sectorial decision making processes. Such communities include the Arabic minority, ultra-orthodox Jews, Ethiopian ethnic group and migrant workers from Africa lacking official status in Israel. For each group, well-phrased messages were delivered taking into account cultural and linguistic barriers as well as direct approach to ensure engagement of community leaders. This attitude proved useful in that vaccine coverage in some of these groups was higher than the coverage in the general population (unpublished data). It is worth mentioning that those communities are far less influenced by the Internet and electronic media.

Many questions arise but still remain unanswered. As public objection to vaccination campaigns as discussed above might increase in the future, there needs to be more engagement of stakeholders in public health decision-making processes. The question is where to draw the line? Is it appropriate to involve non-professionals in a decision making process that is fundamentally professional? Will inclusion of antivaccinist group representatives in the process soften their opposition or just feed information that could be used against vaccination?

The current campaign, by choice, hardly used negative messages about the risks form the WPV 1 , although there were voices suggesting it. Is such 'intimidation' a helpful communication technique when risk perception is distorted in parts of the public?

Can there be too much information in public health? Although much information was delivered throughout the event, unprecedented in volume and in number of media channels involved from an Israeli perspective, it did not stop vaccine opponents and other groups to claim that there was not enough information available'. Noteworthy is that this claim was rejected by the Supreme Court of Justice who dealt with a petition filed by antivaccinists against the current polio SIA. Too much information may cause confusion and misunderstanding, especially when messages are complex. The right 'dose' of information is therefore arguable. One example is the paradoxical effect that messages relating to the risk of vaccine safety and efficacy may cause [3].

Routine communication management, health authorities' general image and availability to the public,

\section{FIGURE 3}

Number of calls to call centre and key events during silent reintroduction of wild-type poliovirus 1 and supplemental immunisation activity with bivalent oral polio vaccine, Israel , 4 August-15 September 2013

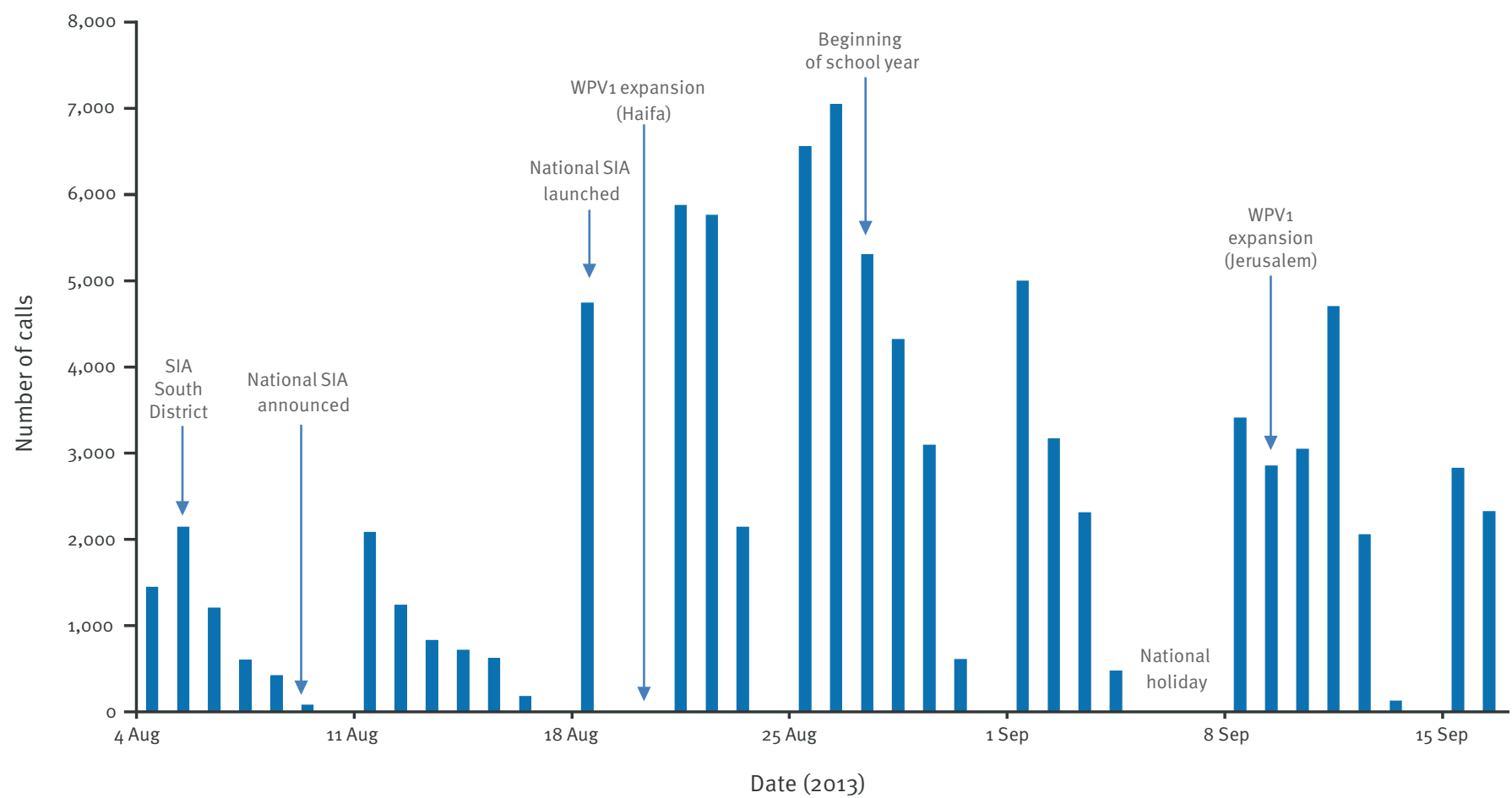

SIA: supplemental immunisation activity; WPV1: wild-type poliovirus 1. 
affects the risk communication in any public health challenge. According to surveys carried out in Israel during the current incident, the level of public trust in the $\mathrm{MoH}$ was high and increased over time. A survey conducted by Government Advertising Bureau in early July 2013 , found that $75 \%$ of respondents thought that the $\mathrm{MoH}$ handled the event properly ( $\mathrm{Y}$ Amikam, personal communication, 10 July 2013). One month later (just before the SIA was launched), this figure rose to $79 \%$.

Interestingly, the links to anti-vaccine sites in the Internet still appear higher up than the $\mathrm{MoH}$ official site in Google searches when typing the word 'polio'. This is exemplified by antivaccinist and $\mathrm{MoH}$ 'YouTube' movies ranking 1st and 10th in Hebrew Google search of 'polio', respectively, despite the fact that the $\mathrm{MoH}$ movie has gained over 200,000 views as compared to only 25,000 for the anti-vaccine movie. Overall, this emphasises the continuous challenge health authorities are facing, even when risk communication is planned thoroughly and performed systematically.

More research that identifies improved methods for communication and sharing of information between public health and healthcare professionals is needed [10]. Communication with professional staff at all levels is critical, and requires planning and sufficient time intervals between transmitting information to professionals and the public, to keep professionals up-todate in parallel to the process of feeding information to the media. Methods for generating uniform and consistent messages, especially when a large number of responders from various disciplines and organisations are involved, also deserve study.

One limitation of our analysis of risk communication is related to its qualitative nature and the fact that exact quantitative measurements and monitoring in an unexpected and untoward situation may be difficult, especially when most resources are invested in response and monitoring other aspects such as clinical and environmental status.

In conclusion, the Israeli $\mathrm{MoH}$ has devised a comprehensive communication strategy in order to facilitate and optimise the various components of the public health response to reintroduction of WPV 1 into Israel, particularly the SIA. Lessons learned from our recent experience may inform risk communication approaches in other countries that may face similar situations as global polio eradication moves towards the 'End game'.

\section{Authors' contributions}

All authors made substantial contributions to conception, design, acquisition and analysis of data. All authors participated in drafting the article or revising it critically for important intellectual content.
Conflict of interest

None declared.

References

1. Anis E, Kopel E, Singer SR, Kaliner E, Moerman L, Moran-Gilad $J$, et al. Insidious reintroduction of wild poliovirus into Israel, 2013. Euro Surveill. 2013;18(38): pii=20586.

2. Hovi T, Shulman LM, van der Avoort H, Deshpande J, Roivainen M, DE Gourville EM. Role of environmental poliovirus surveillance in global polio eradication and beyond. Epidemiol Infect. 2012;140(1):1-13. http://dx.doi.org/10.1017/ So95026881000316X

3. World Health Organization (WHO). Best practices for communicating with the public during an outbreak. Report of the WHO Expert Consultation on Outbreak Communications held in Singapore, 21-23 September 2004. Geneva: WHO. 2005. Available from: http://www.who.int/csr/resources/ publications/WHO_CDS_2005_32web.pdf

4. World Health Organization (WHO). World Health Organization Outbreak Communication Planning Guide. Geneva: WHO. 2008. Available from: http://www.who.int/ihr/elibrary/ WHOOutbreakCommsPlanngGuide.pdf

5. State of Israel Ministry of Health. News and Events. Press Releases. Polio Virus Detected in the Sewerage System of Rahat. 28 May 2013. Available from: http://www.health.gov. il/English/News_and_Events/Spokespersons_Messages/ Pages/28052013_1.aspx

6. Duncan B. How the media reported the first days of the pandemic $\left(\mathrm{H}_{1} \mathrm{~N}_{1}\right)$ 2009: results of EU-wide media analysis. Euro Surveill. 2009;14(30):pii=19286.

7. Betsch C. Innovations in communication: the Internet and the psychology of vaccination decisions. Euro Surveill. 2011;16(17): $\mathrm{pii}=19849$.

8. Kata A. A postmodern Pandora's box: anti-vaccination misinformation on the Internet. Vaccine. 2010;28(7):1709-16. http://dx.doi.org/10.1016/j.vaccine.2009.12.022

9. Baleta AF, van den Heever J, Burnett RJ. Meeting the need for advocacy, social mobilisation and communication in the introduction of three new vaccines in South Africa - successes and challenges. Vaccine. 2012;30 Suppl 3:C66-71. http:// dx.doi.org/10.1016/j.vaccine.2012.06.033

10. Levine H, Balicer RD, Laor D, Grotto I. Challenges and opportunities in the Israeli 2009 pandemic influenza vaccination program. Hum Vaccin. 2011;7(10):1077-82. http:// dx.doi.org/10.4161/hv.7.10.17627

11. Dearinger AT, Howard A, Ingram R, Wilding S, Scutchfield D, Pearce KA, et al. Communication efforts among local health departments and health care professionals during the 2009 H1N1 outbreak. J Public Health Manag Pract. 2011;17(1):45-51. http://dx.doi.org/10.1097/PHH.ob013e3181f54110

12. Polio outbreak in Israel, Dr. Susan Hamphriz revealing the truth about the vaccine. YouTube. 2 Jul 2013. Available from: http:// www.youtube.com/watch?v=UxeGGLi-WEk

13. World Health Organization (WHO). Global Alert and Response (GAR). Poliovirus detected from environmental samples in Egypt. Geneva: WHO. 11 Feb 2013. Available from: http://www. who.int/csr/don/2013_02_11/en/index.html

14. Chen RT. Vaccine risks: real, perceived and unknown. Vaccine. 1999;17 Suppl 3:S41-6. http://dx.doi.org/10.1016/ So264-410X(99)00292-3

15. Maduka $\mathrm{O}$, Tobin-West $\mathrm{Cl}$. Adherence counseling and reminder text messages improve uptake of antiretroviral therapy in a tertiary hospital in Nigeria. Niger J Clin Pract. 2013;16(3):3028. http://dx.doi.org/10.4103/1119-3077.113451

16. Wøien G, Tonsberg KI. Norwegians approve of the health authorities' strategy to communicate worst case pandemic scenarios. Euro Surveill. 2009;14(22):pii=19231.

17. European Commission. Special Eurobarometer 401. Responsible Research and Innovation (RRI), Science and Technology. Brussels: European Commission. Nov 2013. Available from: http://ec.europa.eu/public_opinion/archives/ ebs/ebs_401_sum_en.pdf

18. Taylor S, Shimp L. Using data to guide action in polio health communications: experience from the Polio Eradication Initiative (PEI). J Health Commun. 2010;15 Suppl 1:48-65 http://dx.doi.org/10.1080/10810731003698585

19. Johnson AJ, Mantel P, Aitken P. A strategy for real time improvement (RTI) in communication during the $\mathrm{H}_{1} \mathrm{~N}_{1}$ emergency response. Aust Health Rev. 2010;34(4):493-8. http://dx.doi.org/10.1071/AHo9826 